\title{
Verzeichnis der Abkürzungen
}

\begin{tabular}{|c|c|c|c|}
\hline $\begin{array}{l}\text { AA } \\
\text { AAA }\end{array}$ & $\begin{array}{l}\text { Acta Astronomica } \\
\text { Asociación Argentina de } \\
\text { Astronomia }\end{array}$ & BAB & $\begin{array}{l}\text { Bulletin Astronomique de } \\
\text { l'Observatoire Royal de Bel- } \\
\text { gique }\end{array}$ \\
\hline AAS & American Astronomical Soclety & BAC & Bulletin of the Astronomical \\
\hline AAVSO & $\begin{array}{l}\text { American Association of } \\
\text { Vartable Star Observers }\end{array}$ & BAN & $\begin{array}{l}\text { Institutes of Czechoslovakia } \\
\text { Bulletin of the Astronomical }\end{array}$ \\
\hline Abh. & Abhandlungen & & Institutes of the Netherlands \\
\hline Abstr. & Abstract & BAV Rundbrief & Mitteilungsblatt der Berliner \\
\hline $\begin{array}{l}\text { Abt. } \\
\text { AC }\end{array}$ & $\begin{array}{l}\text { Abtellung } \\
\text { Astronomisches Zirkular der }\end{array}$ & & $\begin{array}{l}\text { Arbeitsgemeinschaft fur Ver- } \\
\text { anderliche Sterne }\end{array}$ \\
\hline & UdSSR & Beob. & Beobachtungen etc. \\
\hline Acad. & Academy etc. & Ber. & Berichte \\
\hline Accod. & émia & BIA & Bulletin des Instituts für \\
\hline AFCRL & $\begin{array}{l}\text { Air Force Cambridge Research } \\
\text { Laboratories }\end{array}$ & & $\begin{array}{l}\text { Theoretische Astronomie, } \\
\text { Leningrad }\end{array}$ \\
\hline $\mathbf{A G}$ & Astronomische Gesellschaft & BMI & Bulletin de Mesures \\
\hline AIAA J & American Institute of Aero- & & Ionosphériques \\
\hline & nautics and Astronautics. & Bol. & Boletín \\
\hline & Journal & BSAF & L'Astronomie. Bulletin de 1 \\
\hline $\mathbf{A J}$ & Astronomical Journal & & Société Astronomiq \\
\hline AJB & Astronomischer Jahresbericht & & France \\
\hline AJ UdSSR & $\begin{array}{l}\text { Astronomisches Journal der } \\
\text { UdSSR }\end{array}$ & $\begin{array}{l}\text { Bull. } \\
\text { Bull hor }\end{array}$ & $\begin{array}{l}\text { Bulletin } \\
\text { Bulletin ho }\end{array}$ \\
\hline Akad. & Akademie & Bull $\mathrm{KE}$ & Bullotin der Stationen \\
\hline $\mathbf{A N}$ & Astronomische Nachrichten & & zur optischen Beobachtung \\
\hline $\begin{array}{l}\text { An. } \\
\text { angew. }\end{array}$ & $\begin{array}{l}\text { Anales etc. } \\
\text { angewandt }\end{array}$ & bzw. & $\begin{array}{l}\text { künstlicher Erdsatelliten } \\
\text { beziehungswelse }\end{array}$ \\
\hline Ann. & Annalen etc. & Ci. & Cie \\
\hline ApJ & Astrophysical Journal & Circ. & Circular etc. \\
\hline Arch. & Ar & Cl. & \\
\hline Ark Astr & Arkiv för Astronoml & Coll. & Collection etc. \\
\hline Aв. & Asociación & Commun. & Communications \\
\hline ASA & $\begin{array}{l}\text { Astronomical Society of Au- } \\
\text { stralia }\end{array}$ & $\begin{array}{l}\text { Comun. } \\
\text { Contr. }\end{array}$ & $\begin{array}{l}\text { Comunicazioni } \\
\text { Contributions etc. }\end{array}$ \\
\hline $\mathbf{A S P}$ & $\begin{array}{l}\text { Astronomical Soclety of the } \\
\text { Paciflc }\end{array}$ & COSPAR IB & $\begin{array}{l}\text { COSPAR Information Bulle- } \\
\text { tin }\end{array}$ \\
\hline $\begin{array}{l}\text { Ass. } \\
\text { Astronaut. }\end{array}$ & $\begin{array}{l}\text { Association } \\
\text { Astronsutik etc. }\end{array}$ & CR & $\begin{array}{l}\text { Comptes Rendus. Académie } \\
\text { des Sciences, Paris }\end{array}$ \\
\hline $\begin{array}{l}\text { Astr. } \\
\text { Astrophys. } \\
\text { Atmosph. }\end{array}$ & $\begin{array}{l}\text { Astronomie etc. } \\
\text { Astrophysik etc. } \\
\text { Atmosphare etc. }\end{array}$ & C.S.I.R.o. & $\begin{array}{l}\text { Commonwealth Scientiflc and } \\
\text { Industrial Research Organi- } \\
\text { zation }\end{array}$ \\
\hline B. & Besprechung(en) & DAN & Berichte (Doklady) der Aka- \\
\hline BA & Bulletin Astronomique & & demie der Wissenschaften de \\
\hline BAA & $\begin{array}{l}\text { British Astronomical Asso- } \\
\text { clation }\end{array}$ & Dep. & $\begin{array}{l}\text { UdSSR, Moskau-Leningrad } \\
\text { Department }\end{array}$ \\
\hline BAA Circ & $\begin{array}{l}\text { British Astronomical Asso- } \\
\text { ciation Circular }\end{array}$ & $\begin{array}{l}\text { dgl. } \\
\text { d. } h .\end{array}$ & $\begin{array}{l}\text { desgleichen } \\
\text { das heißt }\end{array}$ \\
\hline BAAH & $\begin{array}{l}\text { British Astronomical Asso- } \\
\text { ciation Handbook }\end{array}$ & $\begin{array}{l}\text { Diss. } \\
\text { Div. }\end{array}$ & $\begin{array}{l}\text { Dissertation } \\
\text { Division }\end{array}$ \\
\hline
\end{tabular}


EB

Eigenbewegung

Krgebnisse AKE Ergebnisse der Beobachtungen amerikanischer künstlicher Erdsatelliten

Ergebnisse SKE Ergebnisse der Beobachtungen sowjetischer künstlicher Erdsatelliten

ESO

EuW

FHD

Fis.

Gaz astr

gek.

Geod.

Geogr.

Geol.

Geom. Aer.

Geophys.

Ges.

Gravimetr.

Harv Card

Harv Repr

Harv Repr (2)

HeD

HRD

Eydrogr.

Hydrol.

IA U

IBSH

IBVS

ICsU

Industr.

Ing.

Inst.

Ionosph.

J.

J A TP

JBAA

J BIS

JGR

Jo

JOSA
European Southern Observatory

Erde und Weltall

Farben-Helligkeitsdiagramm

Fisica etc.

Gazette astronomique

gekürzt

Geodäsie etc.

Geographie etc.

Geologie etc.

Geomagnetisnuus und Aeronomie

Geophysik etc.

Gesellschaft

Gravimetrie etc.

Harvard College Observatory Announcement Card

Harvard Reprint

Harvard Reprint Series II

Hemel en Dampkring

Hertzsprung-Russell-

Diagramm

Hydrographie etc.

Hydrologie etc.

International Astronomical

\section{Union}

Information Bulletin for the Southern Hemisphere

Information Bulletin on Variable Stars of Commission 27 of the I.A U.

\section{International Council of}

Scientiflc Unions

Industrie etc.

Ingenieur etc.

Institut etc.

Ionosphäre etc.

Journal

Journal of Atmospheric and Terrestrial Physics

Journal of the British Astronomical Association

Journal of the British Interplanetary Societs

Journal of Geophysical

Research

Journal des Observateurs

Journal of the Optical Society

of America

J RAS Canada
KC
KE
Kl.

Journal of the Royal Astronomical Society of Canada Kometenzirkular der Schewtschenko-Universität Kiew Künstliche Erdsatelliten Klasse

Kop Akt Medd Aktuelle Meddelelser fra Astronomisk Selskab, København

Kosm. Forsch. Kosmische Forschungen

Lab.

Mag.

Math.

Mech.

Med.

Medd.

Mém.

Mem.

Mem RAS

Mem SA It

Meteorol.

MIT

Mitt.

MN

MN ASSA

MPC

MVS

Nachr.

Nat.

NAT

Naut.

NBS

(NF)

(NR)

(NS)

Obs.

Obs

Oss.

PAT

Phil.

Phys.

planet.

Proc.

Proc IEEE

Progr.
Laboratorium etc.

Magazine

Mathematik etc.

Mechanik etc.

Mededelingen

Meddelande, Meddelelser

Mémoires

Memoirs, Memorandum etc.

Memoirs of the Royal Astronomical society

Memorie della Societs Astronomica Itallana

Meteorologie etc.

Massachusetts Institute of Technology

Mitteilungen

Monthly Notices of the Royal Astronomical Society

Monthly Notes of the Astronomical Socjety of Southern Africa

Minor Planet Circular Mitteilungen über Verănderliche Sterne, Sonneberg

Nachrichten

Natur etc.

Nordisk Astronomisk Tidsskrift

Nautik etc.

National Bureau of Standards

Neue Folge

Neue Relhe

Neue Serie

Observatorium etc.

The Observatory

Osservatorio, Osservazioni

Populär Astronomisk Tid-

skrift

Philosophical

Physik etc.

planetarisch etc.

Proceedings

Proceedings of the Institute of Electrical and Electronics Engineers

Progress etc. 


\begin{tabular}{|c|c|c|c|}
\hline PTA & $\begin{array}{l}\text { Polskie Towarzystwo Astro- } \\
\text { nomiczne }\end{array}$ & $\begin{array}{l}\text { Suppl. } \\
\text { SuW }\end{array}$ & $\begin{array}{l}\text { Supplement } \\
\text { Sterne und Weltraum }\end{array}$ \\
\hline Pubbl. & Pubblicazioni & $\mathbf{T}$ & Tidskrift etc. \\
\hline Publ. & Publikationen etc. & TAB & Tokyo Astronomical Bulletin \\
\hline Publ ASP & $\begin{array}{l}\text { Publications of the Astrono- } \\
\text { mical Society of the Paciftc }\end{array}$ & $\begin{array}{l}\text { Techn. } \\
\text { TH }\end{array}$ & $\begin{array}{l}\text { Technik etc. } \\
\text { Technische Hochschule }\end{array}$ \\
\hline Rap. & Raportoj & theoret. & theoretisch etc. \\
\hline RAS & Royal Astronomical Scciety & Trans. & ons \\
\hline Rech. & Recherches & t., a. & Ubersetzung, übersetzt \\
\hline Ref. & Referat & U AI Cire & Union Astronomique Inter- \\
\hline Rend. & Rendiconti & & nationale. Circulaire \\
\hline Rep. & Report & UC, Union Cire & Circular of the Union Obser- \\
\hline Repr. & Reprint & & vatory \\
\hline Res. & Research & Univ. & Universitat etc. \\
\hline Rev. & Review etc. & VdS Nachr. & Nachrichtenblatt der Ver- \\
\hline $\mathbf{R G}$ & $\begin{array}{l}\text { Radialgesch windigkelt } \\
\check{R} \text { iš Hyèzd }\end{array}$ & & $\begin{array}{l}\text { einigung der Sternfreunde, } \\
\text { Berlin }\end{array}$ \\
\hline tic. & Ricerche etc. & & $\operatorname{ser}(\mathbf{i n})$ \\
\hline RJ UdSSR & Referatjournal. Astronomie, & Verh. & dlungen \\
\hline & $\begin{array}{l}\text { Geodasie. Akademie der Wis- } \\
\text { senschaften der UdSSR }\end{array}$ & $\begin{array}{l}\text { Veröff. } \\
\text { vgl. }\end{array}$ & thichungen \\
\hline R. 0 . & C.S.I.R.o. & VJS & Vierteljahresschrift \\
\hline Roy. & Royal etc. & VS Bull & Veränderliche Sterne, Bulletin \\
\hline AO & $\begin{array}{l}\text { Smithsonian Institution, } \\
\text { Astrophysical observatory }\end{array}$ & WAGO & $\begin{array}{l}\text { Astronomisch-Geodatische } \\
\text { Gesellschaft der UdSSR }\end{array}$ \\
\hline Scl. & Science etc. & W. B. & Weitere Besprechung(en) \\
\hline Sect. & Section & Wet. & Wetenschappen \\
\hline Ser. & Serie etc. & Wiss. & Wissenschaften \\
\hline S. I. R. & $\begin{array}{l}\text { Service International Rapide } \\
\text { des Latitudes }\end{array}$ & & Zeitschrift \\
\hline Sitzber. & Sitzungsberichte & z. B. & zum Beispiel \\
\hline Sky Tel. & Sky and Telescope & ZD & Zvaigznota Debess \\
\hline Soc. & Society etc. & 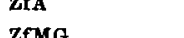 & Zeitschrift für Astrophysik \\
\hline $\begin{array}{l}\text { SSR } \\
\text { Sternw. }\end{array}$ & $\begin{array}{l}\text { Sozialistische Sowjetrepublik } \\
\text { Sternwarte }\end{array}$ & ZfMG & $\begin{array}{l}\text { Zentralblatt für Mathematik } \\
\text { und ihre Grenzgebiete }\end{array}$ \\
\hline 3tud. Cerc. & Studii gi Cercetări & () & (Serie, Folge, Reihe) \\
\hline
\end{tabular}

